\title{
Commissioning the CMS pixel detector with Cosmic Rays
}

\author{
Bernadette Heyburn ${ }^{* \dagger}$ \\ University of Colorado \\ E-mail: heyburnepizero.colorado.edu
}

The Compact Muon Solenoid (CMS) is one of two general purpose experiments at the Large Hadron Collider. The CMS experiment prides itself on an ambitious, all silicon based, tracking system. After almost 20 years of design and construction the CMS tracker detector has been installed and commissioned. The tracker detector consists of ten layers of silicon microstrip detectors while three layers of pixel detector modules are situated closest to the interaction point. The pixel detector consists of 66 million pixels of $100 \mu \mathrm{m} \times 150 \mu \mathrm{m}$ size, and is designed to use the shape of the actual charge distribution of charged particles to gain hit resolutions down to $12 \mu \mathrm{m}$. This paper will focus on commissioning activities in the CMS pixel detector. Results from cosmic ray studies will be presented, in addition to results obtained from the integration of the pixel detector within the CMS detector and various calibration and alignment analyses.

European Physical Society Europhysics Conference on High Energy Physics, EPS-HEP 2009, July 16 - 222009

Krakow, Poland

\footnotetext{
* Speaker.

${ }^{\dagger}$ Thank you to DOE Contract DE-FG02-04ER41290
} 


\section{Introduction}

The Compact Muon Solenoid (CMS) is a general purpose detector built for the LHC to study fundamental properties of particles [1,2]. The CMS Pixel Detector is the innermost subdetector of the CMS experiment. It consists of two parts, the barrel pixels (BPIX) and the forward pixels (FPIX). BPIX consists of three layers at radii $4.3 \mathrm{~cm}, 7.2 \mathrm{~cm}$, and $11.0 \mathrm{~cm}$. FPIX has two disks on either end of the BPIX, at $34.5 \mathrm{~cm}$ and $46.5 \mathrm{~cm}$ from the middle of BPIX (see Figure 1). Each silicon pixel sensor measures $100 \mu \mathrm{m} \times 150 \mu \mathrm{m}$ and is bump-bonded to the ROC [3, 4]. There are a total of approximately $66 M$ pixels. During the Fall of 2008, CMS recorded $\sim 370 M$ events during a Cosmic Run at Four Tesla (CRAFT) (3.8T magnetic field), leading to $\sim 85,000$ tracks with pixel hits.

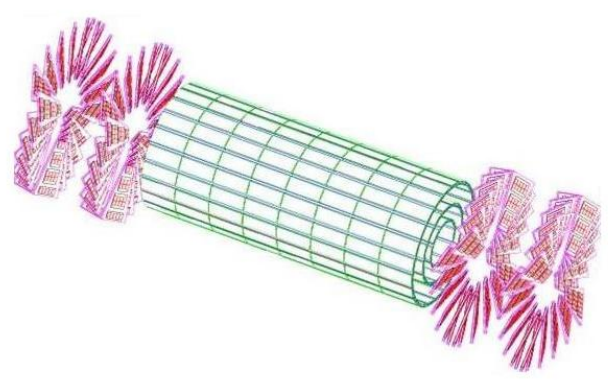

Figure 1: Schematic view of the CMS Pixel Detector with three barrel pixel (BPIX) layers and two forward endcaps (FPIX) on either end.

\section{Calibrations}

The readout thresholds of the pixels influence the hit position resolution. These are measured with an "SCurve Scan". For each pixel the amplitude of an injected test charge (VCAL) is varied from 0 to its maximum. The VCAL value where the signal shows $50 \%$ efficiency is defined as the threshold. The noise is proportional to the width of the region where the signal efficiency switches from 0 to $100 \%$. We measured the BPIX thresholds at $3690 \pm 414$ electrons (averaged at the ROClevel) and the FPIX thresholds as $2870 \pm 194$ electrons, both well below the Minimum Ionizing Partcile (MIP) at $\sim 22,000$ electrons. The noise for the BPIX was measured at $141 \pm 35$ electrons and for the FPIX it was $85 \pm 27$ electrons. This is the internal noise of pixel sensor; the rest of the readout chain typically adds about 300 electrons.

The gain and pedestals of the analog sensors need to be measured to determine cluster charges. The gain is defined as the mean of the slope (units of ADC/VCAL) determined by a linear fit to the signal response function. The pedestal (units of ADC) is the offset of the linear fit. BPIX had an mean gain of $3.324 \pm 0.679$ ADC/VCAL; FPIX had a mean gain of $3.547 \pm .602 \mathrm{ADC} / \mathrm{VCAL}$. The mean BPIX pedestal was measured at $52.59 \pm 22.01$ ADC while the mean FPIX pedestal was measured at $56.43 \pm 11.64 \mathrm{ADC}$. 


\section{Cosmic Run Results}

\subsection{Noisy Pixels}

Noisy pixels are detected by counting the number of events in which a pixel registers a charge above threshold divided by the total number of events, yielding an "event rate." A noisy pixel is defined as having an event rate greater than 0.001. There were 235 noisy pixels in the BPIX and 17 noisy pixels in the FPIX; these pixels were masked during data taking.

\subsection{Pixel Cluster Charge}

The cluster charge is the total charge stored in all adjacet hit pixels. In this study, the cluster charge was corrected for the impact angle and the thickness of the module. For both FPIX and BPIX, the peaks are located around 22000 electrons which fits well with expectations. A comparison of simulation data and CRAFT data was also done. The results of this study are shown in Figure 2.
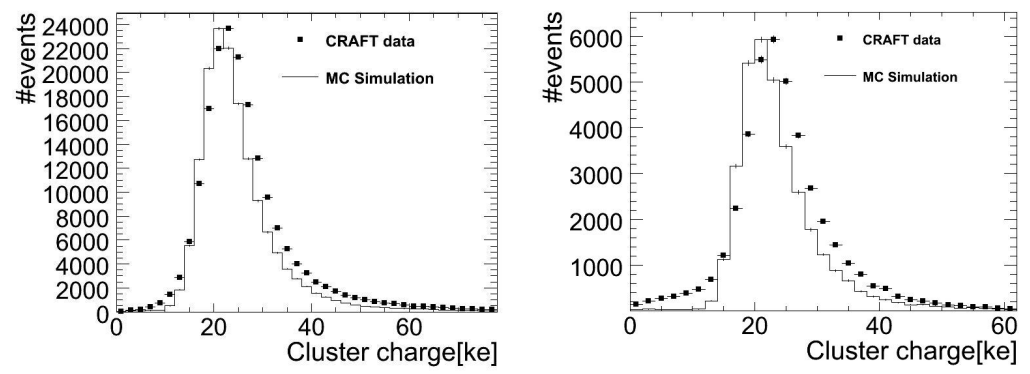

Figure 2: Cluster Charge for CRAFT and MC Data for BPIX (Left) and FPIX (Right)

\subsection{Lorentz Angle Extraction}

The charge carriers are affected by the Lorentz force and are deflected at a Lorentz Angle (LA) with respect to the electric field. The spread of the drifting charge distribution is measured as a function of the track incidence angle which is minimized at the LA. At a magnetic field of $3.8 T$, the value of the LA for the BPIX was determined to be $24.6 \pm 0.1^{\circ}$, and for the FPIX it was determined to be $4.1 \pm 0.3^{\circ}$. The analysis was also performed using data taken while the magnetic field was off; the LA for both the BPIX and FPIX the results were consistent with $0^{\circ}$, which is expected.

\subsection{Barrel Pixel Track Efficiency}

This pixel track efficiency is the ratio of the number of tracks which have at least one valid barrel pixel hit to the subset of tracks that go through the innermost strip tracker barrel and can be propagated through the pixel barrel layers. Results are shown in Figures 3 and 4. The efficiency is low due to the random time structure of the cosmic muons. The pixel detector is sensitive to one clock only. Therefore, the time spread of the cosmic muons results in inefficiencies. With the LHC collisions, the time structure of the pixel hits will be much better defined and therefore we expect the efficiency to be better. 


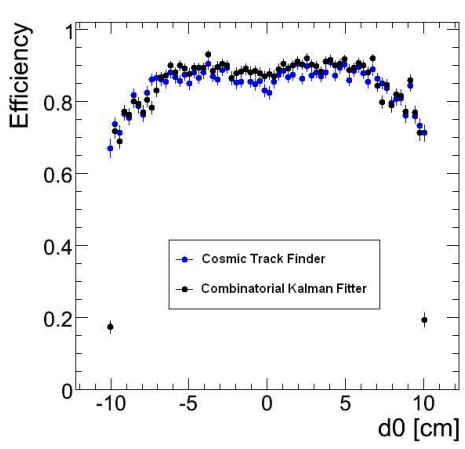

Figure 3: Efficiency as a function of d0, the radial distance from the beam line. The two different CMS tracking algorithms are described here [5].

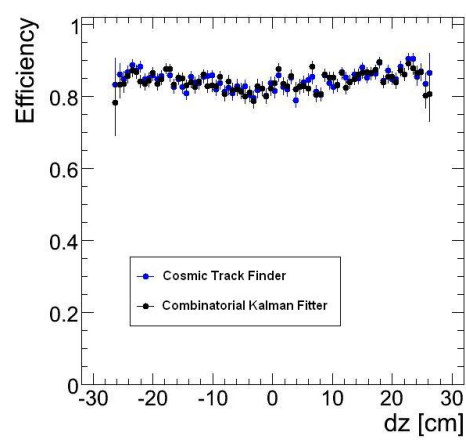

Figure 4: Efficiency as a function of $\mathrm{z}$, the position along the beam line. The two different CMS tracking algorithms are described here [5].

\subsection{Barrel Hit Resolution}

This study uses tracks passing through overlaping modules in the same BPIX layer. For the hit pairs coming from overlapping modules, the hit position and the expected hit position from the track trajectory are compared. The difference in the two hit positions is calculated and also the difference in predicted hit positions and the "double difference" between these two differences is calculated. We expect this to be zero (except for misalignment effects). For each overlap site, the width of this double difference is determined, and it is related to the resolution through $\sigma\left(\Delta X_{h i t}\right)$ : width $h^{2}-\sigma\left(\Delta X_{\text {pred }}\right)^{2}=\sigma\left(\Delta X_{\text {hit }}\right)^{2}$. In the track trajectory, an uncertainty on the position of the track at each module is calculated, then the value of $\sigma\left(\Delta X_{\text {pred }}\right)$ is calculated from the track as well, taking into account the correlations between the positions on the two modules. The resolutions were found to be $19 \pm 2 \mu \mathrm{m}$ in the $\mathrm{X}$ direction, and $31 \pm 3 \mu \mathrm{m}$ in the $\mathrm{Y}$ direction.

\section{Conclusions}

The CMS Pixel detector has been successfully installed within CMS and successfully operated during global runs, in particular CRAFT 2008. The various analyses show that the detector performed well. More calibrations, global runs, and studies are taking place and will take place during Summer and Fall 2009 before we can expect LHC collisions in winter 2009-2010.

\section{References}

[1] CMS Collaboration, CMS Physics TDR Volume 1, CERN-LHCC-2006-001 (2006)

[2] CMS Collaboration, CMS Physics TDR Volume 2, CERN-LHCC-2006-021 (2006)

[3] CMS Collaboration, CMS Tracker Design Report, CERN-LHCC-98-06, CMS-TDR-5 (1998)

[4] CMS Collaboration, Addendum to the CMS tracker TDR, CERN-LHCC-2000-016 (2000).

[5] CMS Tracker Collaboration, W. Adam et al., Stand-alone cosmic muon reconstruction before installation of the CMS silicon strip tracker, JINST 4 P05004 (2009) 